\title{
NEAR CENTRAL AUTOMORPHISMS OF ABELIAN TORSION GROUPS
}

BY

\section{JUTTA HAUSEN}

\begin{abstract}
This paper is concerned with the normal structure of the automorphism group $A(T)$ of an abelian torsion group $T$. The concept of the near center of a group is introduced in order to determine all subgroups of $A(T)$ the centralizer of which has finite index. Consequences are the fact that the finite normal subgroups of $A(T)$ are nilpotent if $T$ is a primary group of infinite rank, and that every normal torsion subgroup of $A(T)$ is contained in the center of $A(T)$ if $T$ is divisible.
\end{abstract}

1. Introduction. The following is trivial: if $S$ is a subgroup of a group $X$ such that $S \leq N \cdot \mathbf{z} X$, where $N$ is a finite normal subgroup of $X$ and $\mathbf{z} X$ denotes the center of $X$, then the centralizer $c S$ of $S$ in $X$ has finite index; this implication however is not reversible.

In this article we will show that for the automorphism group $A(T)$ of an abelian torsion group $T$ the converse in fact is true: if $\Gamma$ is a subgroup of $A(T)$ such that $[A(T): \mathrm{c} \Gamma]$ is finite, then $\Gamma \leq \Delta \cdot \mathrm{z} A(T)$ for some finite normal subgroup $\Delta$ of $A(T)$ [Theorem 6.1]. Moreover, if $T$ is an abelian p-group of infinite rank, then $\Delta$ is a p-group and, hence, $\Gamma$ is nilpotent [Corollary 6.2]. A consequence of this is the fact that, if $G$ is a $p$-group of infinite rank, then all finite normal subgroups $\Gamma$ of $A(G)$ are nilpotent; and $\Gamma \leq \mathrm{z} A(G)$ if $p$ does not divide the order of $\Gamma$. In contrast to that, $\Gamma \leq \mathrm{z} A(G)$ whenever $\Gamma$ is a normal subgroup of $A(G)$ such that $[A(G): \mathrm{c} \Gamma]$ is finite and not divisible by $p-1$ [Theorem 6.4].

These results are easy consequences of our investigations on near central automorphisms in $\$ \$ 4$ and 5: $\gamma \in A(T)$ is called near central, if there exists an integer $k \geq 1$ such that $\gamma \alpha^{k}=a^{k} \gamma$ for all $a \in A(T)$. The set of all near central automorphisms of $T$ is a characteristic subgroup $\mathrm{nz} A(T)$ of $A(T)$ called the near center of $A(T)$. We will show that the near center of $A(T)$ is the product of the center and all finite normal subgroups of $A(T)$. Also, with every $p$-group $G$ there will be associated a finite normal subgroup $\Delta(G)$ of $A(G)$ such that $\mathrm{nz} A(G)=$ $=\Delta(G) \cdot\left(R_{p}^{*} \cdot 1_{G}\right)$, where $R_{p}^{*}$ denotes the group of $p$-adic units. If $G$ has infinite rank, then $\Delta(G)$ will turn out to be a p-group.

Received by the editors June $1,1971$.

AMS (MOS) subject classifications (1970). Primary 20K10, 20K30, 20F 50; Secondary 20F $30,20 \mathrm{~F} 15,20 \mathrm{~F} 55$.

Key words and phrases. Abelian torsion group, automorphism, normal subgroups of automorphism groups, centralizers of subgroups of automorphism groups. 
Basic for our proofs will be a number of rather technical constructions which we have deferred to the "tool section", $\$ 3$. In $\$ 4$ the structure of $p$-groups $G$ for which every near central automorphism is central will be exhibited. We shall show that $G$ possesses a noncentral near central automorphism only if it has the following form: $G=D \oplus B \oplus F$ where $D$ is a divisible group of finite rank, $F$ is finite, and $p^{n} B=0 \neq p^{n} F$ for some integer $n \geq 0$. In particular, $\mathbf{n z} A(G)=\mathbf{z} A(G)$ if $G$ is divisible. This will lead to the description of the near center of $A(T)$ in $\$ 5:$

$$
\mathbf{n z} A(T)=\mathbf{z} A(T) \cdot \coprod_{p}^{\circ} \Delta\left(T_{p}\right)
$$

[Theorem 5.8]. $\$ 6$ contains the cons equences for subgroups $\Gamma$ of $A(T)$ the centralizer of which has finite index in $A(T)$. Besides the results stated above we shall show that every normal torsion subgroup of $A(T)$ is contained in the center of $A(T)$ if $T$ is divisible.

2. Notation and terminology. All groups will be additively written abelian torsion groups, except when groups of automorphisms are under consideration. Mappings are written to the right. Our notation will be standard with possibly the following exceptions: if $T$ is an abelian torsion group, then $d T$ denotes its maximal divisible subgroup, $T_{p}$ its $p$-component and $\mathrm{rk}(T)$ its rank, which is defined as the sum of the ranks of the $T_{p}$. We call $T$ homogeneous if $T$ is the direct sum of pairwise isomorphic subgroups of rank one. If there is no danger of confusion, we may not distinguish between different identity mappings and write 1 instead of $1_{T}$. The group of all automorphisms of $T$ will be denoted by $A(T)$, the ring of endomorphisms of $T$ by $E(T)$. If $\Delta \leq A(T)$, then $\Delta-1$ is the set of all $\delta-1_{T} \in E(T)$, where $\delta \in \Delta$. Our symbols for direct sums are $\oplus$ and $\Sigma^{\circ}$; we will also need symbols for products, direct products, and cartesian products of multiplicative groups, which are $\Pi, \Pi^{\circ}$, and $\Pi^{*}$. The set of all integers is denoted by $l$, the ring of $p$-adic integers by $R_{p}$, and its groups of units by $R_{p}^{*}$.

Furthermore, for $X$ a group and $S$ a subgroup of $X$ :

$$
\begin{aligned}
\mathbf{z} X & =\text { center of } X, \\
\mathbf{n z} X & =\text { near center of } X \text { [see } \S 4], \\
\mathbf{c} S & =\text { centralizer of } S \text { in } X, \\
\mathbf{c}_{T} S & =\text { centralizer of } S \text { in } T \text { where } T \leq X, \\
o(x) & =\text { order of } x \in X, \\
\langle x\rangle & =\text { cyclic subgroup generated by } x, \\
t(X) & =\text { set of all torsion elements of } X, \\
x \circ y & =x^{-1} y^{-1} x y, \\
s \circ y & =\{s \circ y \mid s \in S\},
\end{aligned}
$$




$$
\begin{aligned}
N \triangleleft X & =N \text { is a normal subgroup of } X, \\
Z(n) & =\text { cyclic group of order } n, \\
Z\left(p^{\infty}\right) & =\text { quasi-cyclic } p \text {-group. }
\end{aligned}
$$

If $G$ is an abelian group then $G\left[p^{n}\right]$ denotes the subgroup of $G$ consisting of all elements $x$ such that $p^{n} x=0$. If $A$ is a subgroup of $G$, then the stabilizer of $A$ in $G$ is the set $\Sigma(G: A)$ of all automorphisms of $G$ fixing $A$ elementwise and inducing the identity mapping in $G / A$. It is well known that $\Sigma(G: A) \simeq$ $\operatorname{Hom}(G / A, A)$ (cf. [5, p. 153, 1.4]); in particular, stabilizers are abelian.

3. The tools. Throughout the following, $G$ denotes an abelian $p$-group for some fixed prime $p$. The center $\mathrm{z} A(G)$ of the automorphism group of $G$ has been determined by R. Baer [1, Pp. 110,111]. We list his results for the reader's convenience.

Theorem 3.0 (R. Baer). Let $a$ be an automorphism of G. Then $\alpha=\pi \cdot 1_{G}$ for some p-adic unit $\pi$ if and only if $\alpha$ induces the identity mapping in the lattice of all subgroups of $G$. Moreover, $\mathrm{z} A(G)=R_{p}^{*} \cdot 1_{G}$ if and only if $G$ is not isomorphic to a 2-group $H$ which, for some integer $n \geq 0$, bas the following form:

$$
H=B \oplus Z\left(2^{n+1}\right) \oplus Z\left(2^{\infty}\right), \quad 2^{n} B=0 .
$$

Clearly, $\alpha \in A(G)$ induces the identity mapping in the lattice of all subgroups of $G$ if and only if $x \alpha \in\langle x\rangle$ for all $x \in G$. It is a consequence of the following lemma that, provided $G$ is reduced, $\alpha \in R_{p}^{*} \cdot 1_{G}$ if $x \alpha \in\langle x\rangle$ for all cyclic direct summands $\langle x\rangle$ of $G$.

Lemma 3.1. Let $\epsilon$ be an endomorphism of $G$ such that

(i) $\left.\epsilon\right|_{d G}=\pi \cdot 1_{d G}$ for some p-adic integer $\pi$,

(ii) $x \epsilon \in\langle x\rangle$ for all direct summands $\langle x\rangle$ of $G$. Then $\epsilon=\pi^{\prime} .1_{G}$ for some $\pi^{\prime} \in R_{p}$. If $d G \neq 0$ then $\pi^{\prime}=\pi$.

Proof. Let $G=\mathrm{D} \oplus R$, where $D=d G$ and consider

Case 1. $D \neq 0$. Let $G=\langle x\rangle \oplus C$. Then $D$ contains an element $d$ such that

$$
o(d)=o(x)
$$

and consequently $G=\langle x+d\rangle \oplus C$. By hypothesis there exist $k, l \in I$ such that $x \epsilon=k x,(x+d) \epsilon=l(x+d)$, and we have $d \epsilon=\pi \cdot d$. It follows that $l x+l d=$ $l(x+d)=(x+d) \epsilon=x \epsilon+d \epsilon=k x+\pi \cdot d$ and $l x=k x, l d=\pi \cdot d$. From (1) we obtain $x \epsilon=k x=l x=\pi \cdot x$ whenever $G=\langle x\rangle \oplus C$. If $R$ is bounded, this clearly implies that $\left.\epsilon\right|_{R}=\pi \cdot 1_{R}$ and hence $\epsilon=\pi \cdot 1_{G}$ as claimed. If $R$ is not bounded then for every $r \in R$ there exists a cyclic direct summand $\langle x\rangle$ of $R$ such that $o(x)>o(r)$ and $G=\langle x\rangle \oplus C=\langle x+r\rangle \oplus C$. By what we have shown $x \epsilon=\pi \cdot x$, $(x+r) \epsilon=\pi \cdot(x+r)$, which implies $r \epsilon=\pi \cdot r$ for all $r \in R$ as required. 
Case 2. $D=0$. In this case, of course, $\left.\epsilon\right|_{D}=\pi \cdot 1_{D}$ for all $\pi \in R_{p}$, and, since $G=R$ is reduced, it is enough to show the existence of a $p$-adic integer $\pi$ such that $\left.\epsilon\right|_{B}=\pi \cdot 1_{B}$ for some basic subgroup $B$ of $G$. But this follows readily from the following proposition:

$$
\text { If } G=\langle x\rangle \oplus\langle y\rangle \oplus C \text {, then } x \epsilon=m x \text { and } y \epsilon=m y \text { for some } m \in I \text {. }
$$

In order to prove (2), assume that $o(x) \leq o(y)$. Then also $G=\langle x\rangle \oplus\langle x+y\rangle \oplus C$ and by hypothesis there exist integers $k, l, m$ such that $x \epsilon=k x, y \epsilon=l y,(x+y) \epsilon$ $=m(x+y)$. Consequently, $m x+m y=m(x+y)=(x+y) \epsilon=x \epsilon+y \epsilon=k x+l y$, and hence $x \epsilon=k x=m x, y \epsilon=l y=m y$, proving (2) and completing the proof of this lemma.

The following is technical.

Lemma 3.2. Let $N \geq 1$ be an integer, $g \in G$, and suppose that the basic subgroups of $G$ are unbounded. Then $G=\langle b\rangle \oplus C$, where $g \in C$ and $o(b) \geq N$.

Proof. Since in a p-group without elements of infinite height every element can be imbedded into a finite direct summand (cf. [4, p. 81, 24.8]), $G$ possesses subgroups $F$ and $H$ containing $p^{\omega} G$ such that $G / p^{\omega} G=F / p^{\omega} G \oplus H / p^{\omega} G, g+p^{\omega} G$ $\epsilon F / p^{\omega} G, F / p^{\omega} G$ is finite. It follows that $G=F+H, F \cap H=p^{\omega} G$, and, since $p^{n} G=p^{n} H$ for some $n \in I$, the basic subgroups of $H$ also are unbounded. Therefore, $H$ contains an element $b$ such that $H=\langle b\rangle \oplus H^{\prime}, o(b) \geq N$, for some $H^{\prime} \leq H$. Clearly, since $p^{n} G=p^{n} H$ for sufficiently large $n \in I, p^{\omega} G=p^{\omega} H \leq H^{\prime}$, and $G=$ $F+H=\langle b\rangle+F+H^{\prime}$. Let

$$
k b=f+b \in\langle b\rangle \cap\left(F+H^{\prime}\right), \quad f \in F, b \in H^{\prime} .
$$

Then $f=k b-b \in F \cap H=p^{\omega} G \leq H^{\prime}$, hence $k b=f+b \in H^{\prime} \cap\langle b\rangle=0$, showing that $G=\langle b\rangle \oplus\left(F+H^{\prime}\right)$. By construction, $g \in F \leq F+H^{\prime}=C$, completing the proof.

Lemma 3.3. Let $B$ be a basic subgroup of $G$ and suppose that, for all integers $n \geq 0$, either $p^{n} B$ is infinite or $p^{n} B=0$. Let $y \in G, y \notin p^{\omega} G$, and $k \geq 1$ an integer. Then there exists an automorphism a of $G$ such that $y \alpha^{k} \neq y$.

Proof. Since $y \notin p^{\omega} G$, there exists $b_{1} \in G$ such that $G=\left\langle b_{1}\right\rangle \oplus H$ and $y=$ $m b_{1}+b, b \in H, m b_{1} \neq 0$. In both cases under consideration one verifies [using Lemma 3.2 if $B$ is unbounded] that $G$ contains elements $b_{2}, \cdots, b_{k_{+}}$and a subgroup $C$ such that $G=\left\langle b_{1}\right\rangle \oplus\left\langle b_{2}\right\rangle \oplus \cdots \oplus\left\langle b_{k+1}\right\rangle \oplus C$, o $\left(b_{1}\right) \leq o\left(b_{2}\right) \leq$ $\cdots \leq o\left(b_{k+1}\right)$, and $b \in C$. Let $o\left(b_{i}\right)=p^{e}, i=1, \cdots, k+1$. As shown in [6], the mapping $a$ defined by

$$
\begin{array}{rlrl}
b_{i} \alpha & =b_{i}+p^{e_{i+1}-e_{i}} \cdot b_{i+1} & & \text { for } i=1, \ldots, k, \\
b_{k+1} \alpha & =b_{k+1}, & & \text { for all } c \in C, \\
c \alpha & =c &
\end{array}
$$


extends to an automorphism of $G$, and

$$
b_{1} a^{k}=b_{1}+\sum_{i=1}^{k}\left(\begin{array}{l}
k \\
i
\end{array}\right) p^{e i+1-e_{1}} \cdot b_{i+1} .
$$

Since $o\left(p^{e_{k+1}-e_{1}} \cdot b_{k+1}\right)=o\left(b_{1}\right)$ and $m b_{1} \neq 0$, it follows that $m b_{1} \alpha^{k} \neq m b_{1}$. Hence,

$$
y a^{k}=\left(m b_{1}+b\right) a^{k}=m b_{1} a^{k}+b a^{k} \neq m b_{1}+b=y,
$$

proving the lemma.

Lemma 3.4. Let $B$ be a basic subgroup of $G$ and $k \geq 1$ an integer. If $\eta$ is an endomorphism of $G$ sucb that $G \eta \leq p^{\omega} G$ and $B \eta \neq p B \eta$, then there exists an automorphism a of $G$ such that $\alpha^{k} \eta \neq \eta \alpha^{k}$.

Proof. By hypothesis there exists $w \in B \eta$ such that $B \eta=\langle w\rangle \oplus H, 0 \neq w$. Let $\sigma: B \eta \rightarrow\langle w\rangle$ be the corresponding natural projection onto $\langle w\rangle$. Then $B$ contains an element $b_{1}$ such that $B=\left\langle b_{1}\right\rangle \oplus H_{1}$ and $b_{1} \eta \sigma \neq 0$. Since $B \eta \sigma=\langle w\rangle$ is finite, $H_{1}=F \oplus C$ where $F$ is finite and $C \eta \sigma=0$. It follows from our hypothesis on $B$ that there are $b_{2}, \cdots, b_{k+1} \in B$ such that $b_{i} \eta \sigma=0, i=2, \cdots, k+1$, $G=\left\langle b_{1}\right\rangle \oplus\left\langle b_{2}\right\rangle \oplus \cdots \oplus\left\langle b_{k+1}\right\rangle \oplus H$, and $o\left(b_{1}\right) \leq o\left(b_{2}\right) \leq \cdots \leq o\left(b_{k+1}\right)$. One checks that $G$ possesses an automorphism $a$ satisfying

and that

$$
\begin{aligned}
b_{i+1} \alpha & =b_{i+1}+b_{i} & & \text { for } k \geq i \geq 1, \\
b_{1} \alpha & =b_{1}, & & \\
x \alpha & =x & & \text { for all } x \in H,
\end{aligned}
$$

$$
b_{k+1} a^{k}=b_{k+1}+\sum_{i=0}^{k-1}\left(\begin{array}{c}
k \\
i+1
\end{array}\right) b_{k-i} .
$$

Since $B \eta \leq p^{\omega} G \leq H$ and $\left.\alpha\right|_{H}=1_{H}$, it follows that

$$
\begin{aligned}
b_{k+1} \eta \alpha^{k} \sigma & =b_{k+1} \eta \sigma=0 \neq b_{1} \eta \sigma=b_{k+1} \eta \sigma+\sum_{i=0}^{k-1}\left(\begin{array}{c}
k \\
i+1
\end{array}\right) b_{k-i} \eta \sigma \\
& =\left[b_{k+1}+\sum_{i=0}^{k-1}\left(\begin{array}{c}
k \\
i+1
\end{array}\right) b_{k-i}\right] \eta \sigma=b_{k+1} a^{k} \eta \sigma .
\end{aligned}
$$

Hence, $b_{k+1} \alpha^{k} \eta \neq b_{k+1} \eta \alpha^{k}$ proving the lemma.

Lemma 3.5. Let $G$ be a p-group with basic subgroup $B$ such that, for all integers $n \geq 0$, either $p^{n} B$ is infinite or $p^{n} B=0$. If $\gamma$ is an automorphism of $G$ satisfying, for some integer $k \geq 1$, the condition 


$$
\gamma \circ a^{k}=1_{G} \text { for all } \alpha \in A(G),
$$

then there exists a p-adic integer $\pi$ such that $3\left(\gamma-\pi \cdot 1_{G}\right)$ is divisible.

Proof. Let $G=\langle x\rangle \oplus C$. Then $p^{\omega} G=p^{\omega} C$. Assume that $x y=m x+c, c \in C$, $m \in I$, where $c \notin p^{\omega} C=p^{\omega} G$. Then, by Lemma 3.3, there exists an automorphism $\zeta$ of $C$ such that $c \zeta^{k} \neq c$, which clearly can be extended to an automorphism $a$ of $G$ fixing $x$. Hence

$$
x \alpha^{k} y=x y=m x+c \neq m x+c \zeta^{k}=m x \alpha^{k}+c \alpha^{k}=(m x+c) \alpha^{k}=x y \alpha^{k} .
$$

This contradiction to $(\mathrm{k})$ shows that $x y \equiv m x\left(\bmod p^{\omega} G\right)$ and $x y+p^{\omega} G \in\left\langle x+p^{\omega} G\right\rangle$ for all direct summands $\langle x\rangle$ of $G$. By Lemma 3.1, there exists a $p$-adic integer $\pi$ such that $\left.\gamma\right|_{\left(G / p^{\omega} G\right)}=\pi \cdot 1_{G / p^{\omega} G}$ and hence $G\left(\gamma-\pi \cdot 1_{G}\right) \leq p^{\omega} G$. By hypothesis, $\left(\gamma-\pi \cdot 1_{G}\right) a^{k}=a^{k}\left(\gamma-\pi \cdot 1_{G}\right)$ for all $\alpha \in A(G)$. Applying the contrapositive of Lemma 3.4 for $\eta=\gamma-\pi \cdot 1_{G}$, we obtain that $B\left(\gamma-\pi \cdot 1_{G}\right)$ is divisible as claimed.

4. Near central automorphisms. An element $y$ of a group $X$ is called near central if there exists an integer $k \geq 1$ such that

$$
y x^{k}=x^{k} y \text { for all } x \in X .
$$

One easily verifies that the set of all near central elements of $X$ forms a characteristic subgroup which we shall call the near center of $X$ and denote by $\mathbf{n z} X$. Clearly, the near center contains the center of $X$; if the orders of the elements in $X$ are bounded, then $\mathbf{n z} X=X$. Moreover, if $S$ is a subgroup of $X$ such that the centralizer $\mathrm{c} S$ of $S$ in $X$ has finite index, then $S \leq \mathbf{n z} X$. This follows easily from the fact that a subgroup of $X$ of finite index contains a normal subgroup of $X$ of finite index.

The investigation of near central elements of $A(G)$, where $G$ is an abelian $p$-group, is the subject of this section. The reason why we restrict ourselves to the invertible elements in the endomorphism ring of $G$ becomes clear in view of the following result.

Lemma 4.1. Let the endomorphis $m \eta$ of $G$ satisfy, for some $1 \leq k \in I$, the property $\epsilon^{k} \eta=\eta \epsilon^{k}$ for all $\epsilon \in E(G)$. Then $\eta=\pi \cdot 1_{G}$ for some p-adic integer $\pi$ and bence, $\eta$ is contained in the center of $E(G)$.

Proof. Consider a direct decomposition $G=A \oplus B$ of $G$ and let $\epsilon \in E(G)$ be the corresponding natural projection of $G$ onto $A$. Then $\epsilon^{k}=\epsilon$, and $\epsilon^{k} \eta=\eta \epsilon^{k}$ implies that $a \eta=a \epsilon^{k} \eta=a \eta \epsilon^{k}=a \eta \epsilon$ for all $a \in A$. Consequently,

$$
A \eta \leq A \text { for all direct summands } A \text { of } G \text {. }
$$

Using (3) and the structure theorem for divisible groups, one verifies easily that 
$\left.\eta\right|_{d G}=\pi \cdot 1_{d G}$ for some $\pi \in R_{p}$, and, hence, Lemma 3.1 together with (3) implies $\eta=\pi \cdot 1_{G}$, as claimed.

Definition. An automorphism $\gamma$ of $G$ is called near central if there exists an integer $k \geq 1$ such that

$$
y \circ \alpha^{k}=1_{G} \quad \text { for all } \alpha \in A(G) .
$$

Theorem 4.2. Every near central automorphism of a divisible torsion group is central.

Proof. Clearly, we can restrict ourselves to divisible $p$-groups $D$. Let $\gamma \in \mathbf{n z} A(D)$ and suppose that $\gamma$ is not central. Then, by Theorem $3.0, \gamma$ does not induce the identity mapping in the lattice of all subgroups of $D$. Hence, there exists $d \in D$ such that $d y \notin\langle d\rangle$. Let $D=D_{1} \oplus D_{2} \oplus D_{3}, D_{1} \simeq D_{2} \simeq Z\left(p^{\infty}\right)$, where $d \in D_{1}, d y=d_{1}+d_{2}+d_{3}, d_{i} \in D_{i}$ for $i=1,2,3$, and $d_{2} \neq 0$. By hypothesis, there exists an integer $k \geq 1$ such that

$$
\gamma \circ \alpha^{k}=1_{D} \text { for all } \alpha \in A(D) \text {. }
$$

Since $D_{1}$ is divisible, $d=k d^{\prime}$ for some $d^{\prime} \in D_{1}$, and $d^{\prime} \gamma=d_{1}^{\prime}+d_{2}^{\prime}+d_{3}^{\prime}, d_{i}^{\prime} \in D_{i}$, $k d_{i}^{\prime}=d_{i}$ for $i=1,2,3$. In particular, we have $k d_{2}^{\prime}=d_{2} \neq 0$.

Let $\sigma: D_{2} \rightarrow D_{1}$ be an isomorphism. Then it is easy to verify that $D$ possesses an automorphism $a$ such that

and that

$$
\begin{array}{ll}
x \alpha=x & \text { for } x \in D_{1} \oplus D_{3}, \\
y \alpha=y+y \sigma & \text { for } y \in D_{2},
\end{array}
$$

$$
y a^{k}=y+k y \sigma \quad \text { for all } y \in D_{2} .
$$

Since $\sigma$ is monic and $k d_{2}^{\prime}=d_{2} \neq 0$, it follows that $d_{2}^{\prime} a^{k}=d_{2}^{\prime}+\left(k d_{2}^{\prime}\right) \sigma \neq d_{2}^{\prime}$. Consequently, using $d^{\prime} \in D_{1}$,

$$
\begin{aligned}
d^{\prime} \alpha^{k} \gamma & =d^{\prime} \gamma=d_{1}^{\prime}+d_{2}^{\prime}+d_{3}^{\prime} \\
& \neq d_{1}^{\prime}+d_{2}^{\prime} \alpha^{k}+d_{3}^{\prime}=d_{1}^{\prime} \alpha^{k}+d_{2}^{\prime} \alpha^{k}+d_{3}^{\prime} \alpha^{k}=\left(d_{1}^{\prime}+d_{2}^{\prime}+d_{3}^{\prime}\right) \alpha^{k}=d^{\prime} y \alpha^{k} .
\end{aligned}
$$

This contradiction to $(\mathrm{k})$ proves the theorem.

Corollary 4.3. If $T$ is an abelian torsion group and $\gamma \in A(T)$ is near central, then $\left.\gamma\right|_{d T}$ is central.

Proof. Since $T=d T \oplus C$, every automorphism of $d T$ is induced by some automorphism of $T$. Therefore, the restriction of $y$ to $d T$ is near central and Theorem 4.2 implies the corollary.

Lemma 4.4. Let $G$ be a p-group such that $d G$ bas infinite rank. Then every near central automorphism of $G$ is central. 
Proof. Let $\gamma \in A(G)$ and suppose that, for some $1 \leq k \in I$,

$$
\gamma \circ a^{k}=1_{G} \text { for all } \alpha \in A(G) .
$$

Let $G=D \oplus R, D=d G, \mathrm{rk}(D)$ infinite. By Corollary 4.3 and Theorem 3.0 there exists a $p$-adic integer $\pi$ such that $\left.\gamma\right|_{D}=\pi \cdot 1_{D}$, and without loss of generality we may assume that

$$
\left.\gamma\right|_{D}=1_{D}
$$

Consider $x \in G$ such that $G=D \oplus\langle x\rangle \oplus C$, and $x y=d+m x+c, d \in D, c \in C$, $m \in I$. Suppose $d \neq 0$. Since the rank of $D$ is infinite, $D$ clearly possesses an automorphism $\delta$ such that $d \delta^{k} \neq d$, which can be extended to an automorphism $a$ of $G$ fixing $x$ and $c$. Consequently,

$$
\begin{aligned}
x \alpha^{k} y & =x y=d+m x+c \\
& \neq d \alpha^{k}+m x+c=d \alpha^{k}+m x \alpha^{k}+c \alpha^{k}=(d+m x+c) \alpha^{k}=x y \alpha^{k},
\end{aligned}
$$

contradicting (k). Hence, $x y=m x+c, c \in C$. Assume that $c \neq 0$ and let $D=$ $D_{1} \oplus D_{2}, D_{1} \simeq Z\left(p^{\infty}\right)$. One easily verifies that there exists a homomorphism $\eta: C \rightarrow D_{1}$ such that $c \eta=d_{1} \neq 0$. Since the rank of $D$ is infinite, $D$ possesses an automorphism $\delta$ such that

$$
d_{1}+d_{1} \delta+d_{1} \delta^{2}+\cdots+d_{1} \delta^{k-1} \neq 0
$$

The mapping a defined by

$$
\begin{array}{ll}
x \alpha=x, & \\
y \alpha=y+y \eta & \text { for } y \in C, \\
d \alpha=d \delta & \text { for } d \in D,
\end{array}
$$

can be extended to an automorphism of $G$ and

$$
\begin{aligned}
c \alpha^{k}=\left(c+d_{1}\right) \alpha^{k-1} & =c+d_{1}+d_{1} \alpha+\cdots+d_{1} \alpha^{k-1} \\
& =c+d_{1}+d_{1} \delta+\cdots+d_{1} \delta^{k-1} \neq c
\end{aligned}
$$

by construction. Hence,

$$
x \alpha^{k} y=x y=m x+c \neq m x+c \alpha^{k}=m x \alpha^{k}+c \alpha^{k}=(m x+c) \alpha^{k}=x y \alpha^{k},
$$

again contradicting $(\mathrm{k})$ and proving that $x y \in\langle x\rangle$ for all cyclic direct summands $\langle x\rangle$ of G. Using (4) and Lemma 3.1, it follows that $\gamma$ is central.

Theorem 4.5. Let $G$ be a p-group such that, for all integers $n \geq 0$, either $p^{n} G$ is divisible or $p^{n} G$ bas infinite rank. Then every near central automorphism of $G$ is central.

Proof. Let $G=D \oplus R$ where $D=d G$. By Lemma 4.4 we can assume that the rank of $D$ is finite. Hence, if $B$ is a basic subgroup of $G$, it follows that, 
for all $0 \leq n \in I$, either $p^{n} B$ is infinite or $p^{n} B=0$. Let $\gamma \in A(G)$ be near central. Applying Lemma 3.5, we obtain that there exists a $p$-adic integer $\pi$ such that $B\left(\gamma-\pi \cdot 1_{G}\right)$ is divisible. Hence,

$$
G=D \oplus R=B\left(\gamma-\pi \cdot 1_{G}\right) \oplus D^{\prime} \oplus R
$$

Assume that $B\left(\gamma-\pi \cdot 1_{G}\right) \neq 0$. Since nonzero divisible groups possess automorphisms of arbitrarily high orders, it follows that, for all $1 \leq k \in I$, there exists an automorphism $\beta$ of $B\left(\gamma-\pi \cdot 1_{G}\right)$ such that $\beta^{k} \neq 1$. Hence, $B$ contains an element $b$ such that $G=D \oplus R=D \oplus\langle b\rangle \oplus R^{\prime}, \quad b\left(\gamma-\pi \cdot 1_{G}\right) \beta^{k} \neq b\left(\gamma-\pi \cdot 1_{G}\right)$. Clearly, $\beta$ can be extended to an automorphism $a$ of $G$ fixing $b$. It follows that

$$
b a^{k}\left(\gamma-\pi \cdot 1_{G}\right)=b\left(\gamma-\pi \cdot 1_{G}\right) \neq b\left(\gamma-\pi \cdot 1_{G}\right) \beta^{k}=b\left(\gamma-\pi \cdot 1_{G}\right) a^{k}
$$

and, hence, $b a^{k} \gamma \neq b y a^{k}$, contradicting the near centrality of $\gamma$. Consequently, $B\left(\gamma-\pi \cdot 1_{G}\right)=0$, and $\gamma$ induces a central automorphism in every basic subgroup $B$ of $G$. In particular, $x y \in\langle x\rangle$ for all cyclic direct summands $\langle x\rangle$ of $G$. This together with Corollary 4.3 and Lemma 3.1 implies that $\gamma \in \mathrm{z} A(G)$.

5. The near center of $A(G)$. In order to give a convenient description of nz $A(G)$, we associate with every p-group $G$ a group $\Delta(G)$ of automorphisms of $G$ as follows.

Definition. Let $\Delta(G)=1$ if, for all integers $n \geq 0$, either $p^{n} G$ is divisible or $p^{n} G$ has infinite rank. Otherwise, if $n$ denotes the least nonnegative integer such that $0 \neq\left(p^{n} G\right)[p]$ is finite, then

$$
\Delta(G)=\left\{a \in A(G) \mid G(\alpha-1) \leq p^{n} G \text { and }\left.\alpha\right|_{\left(d G+G\left[p^{n}\right]\right)}=1\right\} .
$$

Clearly, $\Delta(G)$ is a subgroup of $A(G)$.

Theorem 5.1. For every abelian p-group $G, \mathrm{nz} A(G)=\Lambda(G) \cdot\left(R_{p}^{*} \cdot 1_{G}\right)$.

Proof. Suppose first that for all $0 \leq n \in I$ either $p^{n} G$ is divisible or $\left(p^{n} G\right)[p]$ is infinite. Then $\Delta(G)=1$ by definition. Reinhold Baer has shown that, for groups of this structure, $\mathrm{z} A(G)=R_{p}^{*} \cdot 1_{G}$ (Theorem 3.0), and $\mathrm{nz} A(G)=\mathrm{z} A(G$ ) according to our Theorem 4.5. Hence $\mathrm{nz} A(G)=\Delta(G) \cdot\left(R_{p}^{*} \cdot{ }_{1}\right)$, as claimed. Let therefore

$$
G=D \oplus B \oplus F, \quad D=d G, \quad p^{n} B=0 \neq p^{n} F, \quad \text { rk }(D \oplus F) \text { finite },
$$

and either $B=0$ or $p^{n-1} B$ is infinite.

First we want to show that $\mathbf{n z} A(G) \leq \Delta(G) \cdot\left(R_{p}^{*} \cdot 1_{G}\right)$. Suppose that $B=0$. Then $G[p]$ has finite rank and by definition

$$
\Delta(G)=\left\{\alpha \in A(G)|\alpha|_{D}=1\right\}
$$


According to Corollary 4.3, every near central automorphism of $G$ induces in $D$ a central automorphism. Since $\mathrm{z} A(D)=R_{p}^{*} \cdot 1_{D}[1, \mathrm{p} .111]$, it follows that

$$
\mathbf{n z} A(G) \leq\left\{\alpha \in A(G)|\alpha|_{D} \in \mathbf{z} A(D)\right\}=\Delta(G) \cdot\left(R_{p}^{*} \cdot 1_{G}\right)
$$

as claimed. So we may assume that $B \neq 0$, and hence that $p^{n-1} B$ is infinite. Let $\gamma \in \mathbf{n z} A(G)$ satisfy, for some $1 \leq k \in I$, the condition

$$
\gamma \circ \alpha^{k}=1_{G} \text { for all } \alpha \in A(G)
$$

By Corollary 4.3,

$$
\left.\gamma\right|_{D}=\pi \cdot 1_{D} \text { for some } \pi \in R_{p}^{*} \text {. }
$$

Let $x \in F$ and suppose that $x y=d+b+f, d \in D, b \in B, f \in F$, where $b \neq 0$. Lemma 3.3 implies that there exists an automorphism $\delta$ of $D \oplus B$ such that $(d+b) \delta^{k} \neq d+b$, which clearly can be extended to an automorphism $\alpha$ of $G$ fixing $F$ elementwise. Hence

$$
\begin{aligned}
x \alpha^{k} \gamma & =x y=d+b+f \\
& \neq(d+b) \delta^{k}+f=(d+b) \alpha^{k}+f \alpha^{k}=(d+b+f) \alpha^{k}=x y a^{k},
\end{aligned}
$$

contradicting (k). Consequently,

$$
F \gamma \leq D \oplus F
$$

and also $(D \oplus F) \gamma \leq D \oplus F$. It follows that $\gamma$ induces an automorphism $\gamma^{\prime}$ in $G /(D \oplus F)$ which, since every automorphism of $G /(D \oplus F) \simeq B$ is induced by an automorphism of $G$, is near central. Lemma 3.5 implies that $\gamma^{\prime}=\pi^{\prime} \cdot 1$ for some $\pi^{\prime} \in R_{p}^{*}$, and-without loss of generality-we may assume that $\pi^{\prime}=1$, or $G\left(\gamma-1_{G}\right) \leq D \oplus F$. Since $(D \oplus F)\left[p^{n}\right]$ is finite and $\mathrm{p}^{n-1} B$ is infinite, $p^{n} B=0$, it follows that

$$
B=A \oplus C, \quad C \simeq \sum_{\mathcal{K}_{0}}^{\circ} Z\left(p^{n}\right), \quad C(y-1)=0 .
$$

Let $y \in(D \oplus A \oplus F)\left[p^{n}\right]$ and $C=\langle c\rangle \oplus H$ where $o(c)=p^{n}$. Then

$$
G=D \oplus A \oplus C \oplus F=D \oplus A \oplus\langle c+y\rangle \oplus H \oplus F,
$$

and, since $o(c+y)=p^{n}$ and $H \simeq C \simeq \Sigma_{\mathcal{N}_{0}}^{\circ} Z\left(p^{n}\right)$ it is easy to verify that there exists an element $b \in H$ and an automorphism $\alpha$ of $G$ such that $b \alpha^{k}=c+y$. Using $c(\gamma-1)=0=b(\gamma-1)$ and $(\mathrm{k})$ it follows that

$$
c+y=b a^{k}=b y \alpha^{k}=b \alpha^{k} \gamma=(c+y) y=c+y \gamma .
$$

Hence $y \gamma=y$, and we have shown that $(D \oplus A \oplus F)\left[p^{n}\right](\gamma-1)=0$. From (7) together with $C(\gamma-1)=0$, Lemma 3.1 and (5) we obtain that 


$$
\left.\gamma\right|_{\left(d G+G\left[p^{n}\right]\right)}=\pi \cdot 1
$$

We can assume that $F$ has the form

$$
F=\sum_{i=1}^{m}\left\langle x_{i}\right\rangle, \quad o\left(x_{i}\right)>p^{n}
$$

Let $b_{1}, \cdots, b_{m}$ be elements of $B$ such that

$$
B=\sum_{i=1}^{m}\left\langle b_{i}\right\rangle \oplus B^{\prime}, \quad o\left(b_{i}\right)=p^{n}, i=1, \cdots, m .
$$

Then

$$
G=D \oplus B \oplus F=D \oplus B \oplus \sum_{i=1}^{m} \circ\left\langle x_{i}+b_{i}\right\rangle
$$

and, since (6) holds for all direct summands $F$ of $G$ which are complementary to $D \oplus B$, it follows that $F y \leq D \oplus F$ and $F^{\prime} \gamma \leq D \oplus F^{\prime}$, where $F^{\prime}=\sum_{i=1}^{\circ}\left\langle x_{i}+b_{i}\right\rangle$. Let $1 \leq j \leq m$. Then there exist integers $a_{i}, b_{i}, i=1, \cdots, m$, and elements $d_{1}$, $d_{2} \in D$ such that $x_{j} y=\sum_{i=1}^{m} a_{i} x_{i}+d_{1},\left(x_{j}+b_{j}\right) y=\sum_{i=1}^{m} b_{i}\left(x_{i}+b_{i}\right)+d_{2}$. Using (8) we obtain

$$
\sum_{i=1}^{m} b_{i}\left(x_{i}+b_{i}\right)+d_{2}=x_{j} \gamma+\pi \cdot b_{j}=\sum_{i=1}^{m} a_{i} x_{i}+d_{1}+\pi \cdot b_{j},
$$

which implies

$$
\begin{aligned}
& b_{i} x_{i}=a_{i} x_{i} \quad \text { for } i=1, \ldots, m, \\
& b_{i} b_{i}=0 \quad \text { for } i \neq j, \\
& b_{j} b_{j}=\pi \cdot b_{j} .
\end{aligned}
$$

Consequently, $a_{j} \equiv b_{j} \equiv \pi\left(\bmod p^{n}\right)$, and $a_{i} \equiv b_{i} \equiv 0\left(\bmod p^{n}\right)$ for $i \neq j$. It follows that, for $j=1, \cdots, m$,

$$
x_{j} \gamma=\sum_{i=1}^{m} a_{i} x_{i}+d_{1} \equiv \pi \cdot x_{j} \quad\left(\bmod p^{n} G\right),
$$

and hence, that $F\left(\gamma-\pi \cdot 1_{G}\right) \leq p^{n} G$. This together with (8) implies that $G\left(\gamma-\pi \cdot 1_{G}\right) \leq p^{n} G$, and $\gamma=\delta \cdot\left(\pi \cdot 1_{G}\right)$ where $G(\delta-1) \leq p^{n} G$ and $\left.\delta\right|_{\left(d G+G\left[p^{n}\right]\right)}$ $=1$. Hence, $\delta \in \Delta(G)$ and $\gamma \in \Delta(G) \cdot\left(R_{p}^{*} \cdot 1_{G}\right)$, proving nzA(G) $\leq \Delta(G) \cdot\left(R_{p}^{*} \cdot 1_{G}\right)$.

In order to establish the reverse inclusion it suffices to show that $\delta \in \mathbf{n z} A(G)$ for all $\delta \in \Delta(G)$. Let $m \in I$ be such that $F \leq G\left[p^{m}\right]$. Since both $\left(p^{n} G\right)\left[p^{m}\right] \leq$ $D\left[p^{m}\right]+F$ and $G /\left(D+G\left[p^{n}\right]\right) \simeq F / F\left[p^{n}\right]$ are finite and invariant under automorphisms of $G$, there exists an integer $k \geq 1$ such that

$$
\left.a^{k}\right|_{\left(p^{n} G\right)\left[p^{m}\right]}=1 \text { and }\left.\alpha^{k}\right|_{G /\left(D+G\left[p^{n}\right]\right)}=1 \text { for all } \alpha \in A(G) .
$$


We claim that $\Delta(G) \circ \alpha^{k}=1$ for all $\alpha \in A(G)$. Let $\delta \in \Delta(G), \alpha \in A(G)$, and $g \in G$. Since $g\left(\alpha^{k}-1\right) \in D+G\left[p^{n}\right]$ and $\left(D+G\left[p^{n}\right]\right)(\delta-1)=0$, we have $g\left(\alpha^{k}-1\right)(\delta-1)$ $=0$. Clearly, $g(\delta-1)\left(a^{k}-1\right)=0$ if $g \in D \oplus B$. Suppose, that $g \in F$. Then $g(\delta-1) \epsilon\left(p^{n} G\right)\left[p^{m}\right]$, and (9) implies that $g(\delta-1)\left(a^{k}-1\right)=0$. Hence,

$$
g\left(\alpha^{k}-1\right)(\delta-1)=0=g(\delta-1)\left(\alpha^{k}-1\right)
$$

for all $g \in G$, proving $\left(a^{k}-1\right)(\delta-1)=0=(\delta-1)\left(a^{k}-1\right)$ and $a^{k} \delta=\delta a^{k}$ for all $a \in A(G)$ and all $\delta \in \Delta(G)$. It follows that $\Delta(G) \leq \mathbf{n z} A(G)$, completing the proof.

Lemma 5.2. Let $1 \leq n \in I$, and let

$$
\Delta=\left\{\alpha \in A(G) \mid G(\alpha-1) \leq p^{n} G \text { and }\left.\alpha\right|_{\left(d G+G\left[p^{n}\right]\right)}=1\right\} \text {. }
$$

If $p^{n} G$ has finite rank, then $\Delta$ is a finite normal $p$-subgroup of $A(G)$.

Proof. The normality follows from the fact that $p^{n} G$ and $d G+G\left[p^{n}\right]$ both are characteristic subgroups of $G$. By hypothesis,

$$
G=D \oplus B \oplus F, \quad D=d G, \quad p^{n} \bar{B}=0, \quad \operatorname{rk}(D \oplus F) \text { finite }
$$

Since $B \leq G\left[p^{n}\right]$ and $\left.\Delta\right|_{B}=1$, every automorphism in $\Delta$ induces an automorphism $H=G / B \simeq D \oplus F$, and from $G(\Delta-1) \leq p^{n} G$ and $p^{n} G \cap B=0$ it follows that only the 1-automorphism will induce the identity mapping in $H$. Hence, if we put $\Psi=\left.\Delta\right|_{H},\left.\Delta \simeq \Delta\right|_{H}=\Psi, H(\Psi-1) \leq p^{n} H$. Let $\Sigma$ be the set of all $\psi \in \Psi$ inducing the identity automorphism in $p^{n} H$. Then $\Sigma$ is a normal subgroup of $\Psi$, the restriction of $\Psi$ to $p^{n} H$ is isomorphic to $\Psi / \Sigma$, and $\Sigma$ is contained in the stabilizer $\Sigma\left(H: p^{n} H\right)$ of $p^{n} H$ in $H$. It is well known that $\Sigma\left(H: p^{n} H\right) \simeq \operatorname{Hom}\left(H / p^{n} H, p^{n} H\right)$ (cf. [5, p. 153]). Since $H \simeq D \oplus F$ has finite rank it follows that $\operatorname{Hom}\left(H / p^{n} H, p^{n} H\right)$ is a finite $p$-group and, hence, so is $\Sigma$. Therefore, the proof will be completed once we show that $\Psi /\left.\Sigma \simeq \Psi\right|_{p^{n} H}$ is a finite $p$-group. If $p \neq 2,3$ this is a consequence of much deeper results by Freedman [3] and Leptin [8]. In our special case we simply proceed by complete induction on the exponent of $G / d G$ : if $p G=$ $d G$, then $\Delta=1$ and our lemma holds true. Suppose, it is true for all groups $K$ such that the exponent of $K / d K$ is less than the exponent of $G / d G$. One verifies easily that an automorphism inducing the identity in $H / p^{n} H$ also induces the identity mapping in $p^{n} H / p^{2 n} H$. Hence, for $K=p^{n} H$,

$$
\left.\Psi\right|_{K} \leq\left\{\alpha \in A(K) \mid K(\alpha-1) \leq p^{n} K \text { and }\left.\alpha\right|_{\left(d K+K\left[p^{n}\right]\right)}=1\right\} \text {. }
$$

Since the exponent of $K^{\prime} d K=p^{n} H{ }^{\prime} d H \simeq p^{n} F$ is less than the exponent of $G / d G$, we may apply our induction hypothesis and obtain that $\left.\Psi\right|_{K}=\left.\Psi\right|_{p^{n} H} \simeq \Psi / \Sigma$ is a finite $p$-group, completing the proof of this lemma.

Lemma 5.3. For $G$ a p-group, $\Delta(G)$ is a finite normal subgroup of $A(G)$. If the rank of $G$ is infinite then $\Delta(G)$ is a p-group and bence nilpotent. 
Proof. The normality is clear. If $G$ has infinite rank then either $\Delta(G)=1$ or $\Delta(G)$ is a finite p-group according to the definition of $\Delta(G)$ and the previous result. Suppose that the rank of $G$ is finite, $G=D \oplus F, D=d G, G[p]$ finite. Then, by definition,

$$
\Delta(G)=\left\{\alpha \in A(G)|\alpha|_{D}=1\right\}
$$

Let $\Sigma$ be the set of all $\delta \in \Delta(G)$ inducing the identity mapping in $G / D \simeq F$. Again, $\Sigma$ is a normal subgroup of $\Delta(G)$ and $\Delta(G) / \Sigma$ is isomorphic to the group of automorphisms of $G / D$ induced by $\Delta(G)$. Since $G / D \simeq F$ is finite this implies the finiteness of $\Delta(G) / \Sigma$. So, only the finiteness of $\Sigma$ remains to be shown. By construction, $\Sigma$ coincides with the stabilizer $\Sigma(G: D)$ of $D$ in $G$, and

$$
\Sigma=\Sigma(G: D) \simeq \operatorname{Hom}(G / D, D)
$$

(cf. [5, p. 153]). Since, if $m \geq 1$ is an integer such that $p^{m} F=0$ then

$$
\operatorname{Hom}(G / D, D) \simeq \operatorname{Hom}\left(F, D\left[p^{m}\right]\right),
$$

and $D\left[p^{m}\right]$ is finite, $\operatorname{Hom}(G / D, D)$ is finite and so is $\Sigma$. This completes the proof.

Corollary 5.4. If $G$ is a p-group of infinite rank, then the near center of $A(G)$ is nilpotent.

Proof. Theorem 5.1 and Lemma 5.3.

Theorem 5.5. Let $G$ be a p-group and $\Gamma$ a normal subgroup of $A(G)$. Then the following conditions are equivalent.

(i) $A(G) / \mathrm{c} \Gamma$ is finite.

(ii) $\Gamma \leq \mathbf{n z} A(G)$.

(iii) $\Gamma \leq \Delta \cdot\left(R_{p}^{*} \cdot 1_{G}\right)$ for some finite normal subgroup $\Delta$ of $A(G)$.

(iv) $\Gamma \leq \Delta \cdot \mathrm{z} A(G)$ for some finite normal subgroup $\Delta$ of $A(G)$.

Proof. The proof is cyclic, using Theorem 5.1 together with Lemma 5.3 and $R_{p}^{*} \cdot 1_{G} \leq \mathrm{z} A(G)$, and is left to the reader.

Corollary 5.6. Let $G$ be a p-group. Then $A(G) / \mathrm{cnz} A(G)$ is finite; nz $A(G)$ is the set the oretical union of all subgroups $S$ of $A(G)$ such that $[A(G): \mathrm{c} S]$ is finite; if $\pi$ denotes the set of all finite normal subgroups $\Gamma$ of $A(G)$, then also

$$
\mathbf{n z} A(G)=\left(\prod_{\Gamma \in \pi} \Gamma\right)\left(R_{p}^{*} \cdot 1_{G}\right)=\mathbf{z} A(G) \cdot\left(\prod_{\Gamma \in \pi} \Gamma\right) .
$$

We close this section with a description of the near center of $A(T)$, where $T$ is a n abelian torsion group. For this we need the following result which is technical. 
Lemma 5.7. Let $G$ be a p-group and $\gamma \in A(G)$ such that, for some integer $k \geq 1$,

$$
\gamma \circ a^{k}=1 \text { for all } \alpha \in A(G) .
$$

If $p-1$ does not divide $k$, then $\gamma$ is contained in the center of $A(G)$.

Proof. Since $\gamma \in \mathbf{n z} A(G)$, its restriction to $d G$ is a central automorphism according to Corollary 4.3. Applying Lemma 3.1, it therefore suffices to show that $x y \in\langle x\rangle$ if $G=\langle x\rangle \oplus C$. Suppose that $x y=m x+c, m \in I, c \in C$. Since $p-1$ does not divide $k$ there exists an integer $n$ such that $0 \not \equiv n^{k} \not \equiv 1(\bmod p)$. Clearly, $G$ possesses an automorphism $\alpha$ such that

$$
\begin{aligned}
& x \alpha=x, \\
& y \alpha=n y \quad \text { for all } y \in C,
\end{aligned}
$$

and hence, $c \alpha^{k}=n^{k} \cdot c$. It follows that

$$
m x+c=x y=x \alpha^{k} y=x y \alpha^{k}=(m x+c) \alpha^{k}=m x+c \alpha^{k}=m x+n^{k} \cdot c,
$$

which implies

$$
c=n^{k} \cdot c
$$

Since $n^{k} \not \equiv 1(\bmod p)$, we obtain $c=0$ and $x y=m x \in\langle x\rangle$ concluding the proof.

Contrary to the center, the near center of $A(T)$ is not the cartesian product of the near centers of the $A\left(T_{p}\right)$.

Theorem 5.8. Let $T=\Sigma_{p}^{\circ} T_{p}$ be an abelian torsion group. Then

$$
\mathbf{n z} A(T)=\mathbf{z} A(T) \prod_{p}^{\circ} \mathbf{n z} A\left(T_{p}\right)=\prod_{p}^{\circ} \Delta\left(T_{p}\right) \cdot \prod_{p}^{*}\left(R_{p}^{*} \cdot 1_{T}\right) .
$$

Proof. Provided the customary imbeddings and identifications, it is clear that $\Pi_{p}^{\circ} \mathbf{n z} A\left(T_{p}\right) \leq \mathbf{n z} A(T)$. Hence

$$
\begin{aligned}
\mathrm{nz} A(T) & \geq \mathrm{z} A(T) \cdot \prod_{p}^{\circ} \mathrm{nz} A\left(T_{p}\right)=\mathrm{z} A(T) \cdot \prod_{p} \circ \Delta\left(T_{p}\right) \\
& =\prod_{p}^{\circ} \Delta\left(T_{p}\right) \cdot \prod_{p}^{*} \mathrm{z} A\left(T_{p}\right)=\prod_{p}^{\circ} \Delta\left(T_{p}\right) \cdot \prod_{p}^{*}\left(R_{p}^{*} \cdot 1_{T_{p}}\right),
\end{aligned}
$$

using Theorem 3.0 and the fact that $\mathrm{z} A\left(T_{p}\right) \leq \mathbf{n z} A\left(T_{p}\right)=\Delta\left(T_{p}\right) \cdot\left(R_{p}^{*} \cdot 1_{T_{p}}\right)$.

In order to establish the reverse inclusion, let $\gamma \in \mathrm{nz} A(T)$ and suppose that, for some $1 \leq k \in I$,

$$
y \circ \alpha^{k}=1 \text { for all } \alpha \in A(T) \text {. }
$$

Let $\gamma_{p}=\left.\gamma\right|_{T_{p}}$. Clearly, $\gamma_{p} \in \mathbf{n z} A\left(T_{p}\right)$ for all $p$. Since $k$ is fixed, there exist only finitely many primes $p$ such that $p-1$ divides $k$. Hence, by Lemma 5.7, $\gamma_{p}$ is a central automorphism of $T_{p}$ for almost all $p$. It follows that 


$$
\gamma=\zeta \cdot \delta, \quad \zeta \in \prod_{p}^{*} \mathrm{z} A\left(T_{p}\right)=\mathrm{z} A(T), \quad \delta \in \prod_{p}^{\circ} \mathrm{nz} A\left(T_{p}\right)
$$

concluding the proof.

6. Some consequences. Let $T$ again be an abelian torsion group.

Theorem 6.1. If $\Gamma$ is a subgroup of $A(T)$ such that $[A(T): \mathrm{c} \Gamma]$ is finite, then $\Gamma \leq \Delta \cdot \mathrm{z} A(T)$ for some finite normal subgroup $\Delta$ of $A(T)$. Moreover, if $\Gamma$ induces central automorphisms in all p-components of finite rank, then $\Delta$ and bence $\Gamma$ is nilpotent.

Proof. Let $\Gamma_{p}=\left.\Gamma\right|_{T_{p}}$ and again, let us identify an automorphism $\delta$ of $T_{q}$ with the automorphism $\alpha$ inducing $\delta$ in $T_{q}$ and fixing all other $p$-components elementwise. Then

and

$$
\Gamma \leq \prod_{p}^{*} \Gamma_{p} \leq \prod_{p}^{*} A\left(T_{p}\right)=A(T)
$$

$$
\mathrm{c} \Gamma=\mathrm{c}\left(\prod_{p}^{*} \Gamma_{p}\right)=\prod_{p}^{*}\left(\mathrm{c}_{A\left(T_{p}\right)} \Gamma_{p}\right) .
$$

The finiteness of $[A(T): \mathrm{c} \Gamma]$ implies that $\left[\Pi^{*}{ }_{p} A\left(T_{p}\right): \Pi_{p}^{*}\left(\mathbf{c}_{A\left(T_{p}\right)} \Gamma_{p}\right)\right]$ is finite. Hence $\left[A\left(T_{p}\right): \mathbf{c}_{A\left(T_{p}\right)} \Gamma_{p}\right]=1$ for almost all $p$. Let $\Gamma_{p} \nless \mathbf{z} A\left(T_{p}\right)$ if and only if $p \in\left\{p_{1}, \cdots, p_{n}\right\}$. Clearly, $\Gamma_{p} \leq \mathbf{n z} A\left(T_{p}\right)$ for all $p$, and therefore, using Theorem 5.1 and Lemma 5.3, $\Gamma_{p_{i}} \leq \Lambda\left(T_{p_{i}}\right) \cdot \mathrm{z} A\left(T_{p_{i}}\right), i=1, \cdots, n$, where $\Delta\left(T_{p_{i}}\right)$ is a finite normal subgroup of $A\left(T_{p_{i}}\right)$, even a $p_{i}$-group if $T_{p_{i}}$ has infinite rank. It follows that

$$
\lrcorner=\prod_{i=1}^{n} \Delta\left(T_{p_{i}}\right)
$$

is a finite normal subgroup of $A(T)$ which is nilpotent if $\Gamma$ induces central automorphis ms in all $p$-components of finite rank. Hence

$$
\Gamma \leq \prod_{p}^{*} \Gamma_{p} \leq \prod_{i=1}^{n} \Delta\left(T_{p_{i}}\right) \cdot \prod_{p}^{*} \mathrm{z} A\left(T_{p}\right)=\Delta \cdot \mathbf{z} A(T),
$$

proving the theorem.

Corollary 6.2. Let $G$ be an abelian p-group of infinite rank and $\Gamma$ a subgroup of $A(G)$ such that $[A(G): \mathrm{c} \Gamma]$ is finite. Then $\Gamma \leq \Delta \cdot \mathrm{z} A(G)$ for some finite normal p-subgroup $\Delta$ of $A(G)$.

Corollary 6.3. Let $G$ be as in 6.2. Then every finite normal subgroup $\Gamma$ of $A(G)$ is nilpotent. Moreover, if $p$ does not divide the order of $\Gamma$, then $\Gamma$ is contained in the center of $A(G)$. 
Proof. Corollary 6.2.

Theorem 6.4. Let $G$ be a p-group and $\Gamma$ a normal subgroup of $A(G)$ such that $A(G) / \mathrm{c} \Gamma$ is finite. If $p-1$ does not divide the order of ${ }^{*} A(G) / \mathrm{c} \Gamma$ then $\Gamma \leq$ $\mathrm{z} A(G)$.

Proof. If $k$ is the order of $A(G) / \mathrm{c} \Gamma$, then $\gamma \circ \alpha^{k}=1$ for all $\alpha \in A(G)$ and all $\gamma \in \Gamma$. Apply Lemma 5.7.

We conclude with a result on normal torsion subgroups of $A(D)$, where $D$ is a divisible torsion group.

Theorem 6.5. Every normal torsion subgroup of $A(D)$ is contained in the center of $A(D)$.

Proof. Clearly, we may restrict ourselves to the case where $D$ is a $p$-group. Let $\Gamma$ be a normal torsion subgroup of $A(D)$, let $y \in \Gamma$ and $x, y \in D$. One verifies, using heavily the fact that $\gamma$ is a torsion element, that $D$ contains subgroups $D_{1}$ and $C$ such that $D=D_{1} \oplus C, x, y \in D_{1}, D_{1} y=D_{1}$ and

$$
D_{1} \text { has finite rank. }
$$

Let $\Phi$ denote the set of all $\alpha \in A(D)$ such that $D_{1} a=D_{1}$, and let $\Sigma$ be the set of all $\phi \in \Phi$ fixing $D_{1}$ elementwise. Then $\gamma \in \Phi$, furthermore, $\Sigma$ is a normal subgroup of $\Phi$, and $\Phi /\left.\Sigma \simeq \Phi\right|_{D_{1}}$. Since every automorphism of $D_{1}$ is induced by some automorphism of $D$, it follows that $\Phi / \Sigma \simeq A\left(D_{1}\right)$. Consequently, $[(\Phi \cap \Gamma) \Sigma] / \Sigma$ is isomorphic to a normal torsion subgroup $\Gamma_{1}$ of $A\left(D_{1}\right)$ :

$$
[(\Phi \cap \Gamma) \Sigma] / \Sigma \simeq \Gamma_{1} \triangleleft A\left(D_{1}\right), \quad \Gamma_{1} \text { torsion. }
$$

We apply a theorem of $\mathrm{R}$. Baer which states that every torsion group of automorphisms of an abelian torsion group of finite rank is finite [2, p. 521]. Hence, (10) implies that $\Gamma_{1}$ is finite, and therefore so is $A\left(D_{1}\right) / \mathbf{c} \Gamma_{1}$. Since according to Theorem 4.2, $\mathrm{nz} A\left(D_{1}\right)=\mathrm{z} A\left(D_{1}\right)$, it follows that $\Gamma_{1}$ is central which, since $D_{1}$ is divisible, is equivalent to $\Gamma_{1} \leq R_{p}^{*} \cdot 1_{D_{1}}$ (Theorem 3.0). By construction, $\gamma \in \Phi \cap \Gamma$, so that $\left.y\right|_{D_{1}} \in \Gamma_{1}$, and $x, y \in D_{1}$. Hence

$$
x y=\pi \cdot x, \quad y y=\pi \cdot y
$$

for some $p$-adic unit $\pi$. This being true for all $x, y \in D$ and all $\gamma \in \Gamma$ proves the theorem.

\section{REFERENCES}

1. R. Baer, Primary Abelian groups and their automorphisms, Amer. J. Math. 59 (1937), 99-117.

2. - Finite extensions of Abelian groups with minimum conditions, Trans. Amer. Math. Soc. 79 (1955), 521-540. MR 17, 125.

3. H. Freedman, The automorphisms of countable primary reduced Abelian groups, Proc. London Math. Soc. (3) 12 (1962), 77-99. MR 24 \#A3215. 
4. L. Fuchs, Abelian groups, Publ. House Hungarian Acad. Sci., Budapest, 1958. MR $21 \# 5672$.

5. J. Hausen, Automorphismengesättigte Klassen abzählbarer abelschen Gruppen, Sympos. Studies on Abelian Groups (Montpellier, 1967), Springer, Berlin, 1968, pp. 147181. MR 39 \#5691.

6. - Abelian torsion groups with artinian primary components and their automorphisms, Fund. Math. 71 (1971), 273-283.

7. - Automorphisms of Abelian torsion groups with finite p-ranks, Arch. Math. 52 (1971), 128-135.

8. H. Leptin, Eilige Bemerkungen über die Automorphismen Abelscher p-Gruppen, Proc. Colloq. Abelian Groups (Tihany, 1963), Akad. Kiadó, Budapest, 1964, pp. 99-104. MR 29 \#5929.

DEPARTMENT OF MATHEMATICS, UNIVERSITY OF HOUSTON, HOUSTON, TEXAS 77004 